\title{
Theoretical Evaluation of the Electrophilic Catalyses in Successive Enolization and Reketonization Reactions by $\Delta^{5}$-3-Ketosteroid Isomerase
}

\author{
Hwangseo Park, Junghun Suh, and Sangyoub Lee \\ School of Chemistry and Molecular Engineering, and Center for Molectlar Catalysis, \\ Seoul National University; Seoul 151-747, Korea \\ Received Jamary: 28, 2002
}

\begin{abstract}
Based on ab initio calculations at the MP2(FULL)/6-31-G**/RHF/6-31G** level, we compare the energetic and mechanistic features of a model reaction for catalytic action of $\Delta^{5}$-3-ketosteroid isomerase (KSI, E.C. 5.3.3.1) with those of a corresponding nonenzymatic reaction in aqueous solution. The results show that the two catalytic acid residues. Tyr 4 and Asp99, can lower the free energy of activation by $8.6 \mathrm{kcal} / \mathrm{mol}$, which is in good agreement with the experimentally predicted $\sim 9 \mathrm{kcal}$ mol contribution of electrophilic catalyses to the whole enzymatic rate enhancement. The dienolate intenmediate formed by proton transfer from the substrate carbon acid to the catalytic base residue (Asp38) is predicted to be stabilized by $12.0 \mathrm{kcal} / \mathrm{mol}$ in the enzymatic reaction, making its formation thermodynamically favorable. It has been argued that enzymes catalyzing the reactions of carbon acids should resolve the thermodynamic problem of stabilizing the enolate intermediate as well as the kinetic problem of lowening the free energy of activation for proton abstraction. We find that KSI can successfully overcome the thermodynamic difficulty inherent in the nonenzymatic reaction through the electrophilic catalyses of the two acid residues. Owing to the stabilization of dienolate intermediate, the reketonization step could influence the overall reaction rate more significantly in the KSI-catalyzed reaction than in the nonenzymatic reaction, further supporting the previous expenimental findings. However, the electrophilic catalyses alone cannot account for the whole catalytic capability (12-13 kcal $/ \mathrm{mol})$, confirming the earlier experimental implications for the involvement of additional catalytic components. The present computational study indicates clearly how catalytic residues of KSI resolve the fundamental problems associated with the entropic penalty for forming the rate-limiting transition state and its destabilization in the bulk solvation environment.
\end{abstract}

Keywords : $A b$ initio study of enzyme reactivity, $\Delta^{5}-3$-Ketosteroid isomerase.

\section{Introduction}

The proton abstraction from carbon acids, which is a key step in various enzyme-catalyzed reactions, is inherently slow compared to that from oxygen and nitrogen acids. For example, the deprotonation of carbonyl $\alpha$-carbons with $\mathrm{p} K_{\mathrm{a}}$ 's $10-13$ by the hydroxide ion occurs at a rate more than $10^{7}$-fold slower than that for oxygen or nitrogen acids with the same $\mathrm{p} K_{\mathrm{a}}{ }^{1}$ An imbalance in the transition state, characterized by time-lag of resonance stabilization of the incipient carbanionic transition state behind the proton transfer, has been widely invoked to explain such an exceptionally low reaction rate. ${ }^{2}$ Indeed, the transition state imbalance has been shown to be a general phenomenon for proton abstraction from carbon acids that involve delocalization of negative charge into a $\pi$-acceptor group. ${ }^{3}$

A number of enzymes such as isomerase, racemase, tautomerase, and synthase are able to accelerate the proton abstraction from the $\alpha$-carbon of a carbonyl compound. Of these, 3-oxo- $\Delta^{5}$-steroid isomerase ( $\Delta^{*}$-3-ketosteroid isomerase, KSI, E.C. 5.3.3.1) has served as a paradigm for enzymatic enolizations since it acts on its substrate at a rate that

"To whom correspondence should be addressed. Phone: +82-2875-4887; Fax: +82-2-874-3704; E-mail: sangyoubasnu.ac.kr approaches the diffusion-controlled limit. ${ }^{4}$ This enzyme catalyzes the tautomerism of $\beta, \gamma$ and $\alpha, \beta$-unsaturated ketosteroids via a dienolate intermediate. Earlier crystallographic and enzymological studies indicated that the catalysis proceeds through the abstraction of an $\alpha$-proton from the substrate by Asp 38 and the simultaneous stabilization of a negative charge developed on the carbonyl oxygen by the hydrogen bond from Tyr14, followed by the reketonization of the resulting dienolate intermediate by protonated Asp 38 . $^{5}$ Thus, the concerted general acid-base catalysis by Tyr14 and Asp38 was the generally accepted mechanistic model for the KSI action.

However, there is also some experimental evidence suggesting that an additional unknown residue with $\mathrm{p} K_{\mathrm{u}}>$ 8.5 should be involved in the catalytic mechanism of KSI. ${ }^{6}$ A few years ago, Wu and coworkers pointed out the importance of the active site residue, Asp99, in the catalytic reaction. ${ }^{7}$ On the basis of the solution structure combined with the kinetics, mutagenesis, and inhibitor binding studies they proposed that, as depicted in Scheme 1, Asp99 as well as Tyr14 would donate a hydrogen bond to the substrate carbonyl oxygen, thereby stabilizing the negative charge developed on this atom during the catalytic cycle.

This mechanistic aspect of KSI has been further confirmed by several studies based on multidimensional NMR and $\mathrm{X}$-ray crystallographic methods, ${ }^{8}$ although an alternative 

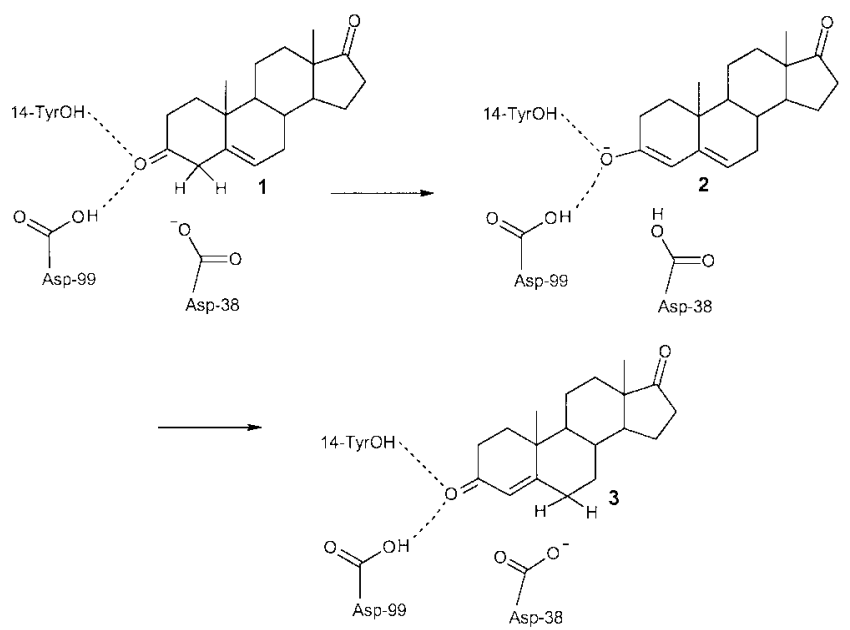

Scheme 1

mechanism (namely the hydrogen-bonded catalytic diad mechanism) in which Asp99 forms a hydrogen bond with phenolic oxygen of Tyrl4 was also suggested. ${ }^{9}$ Recently, Kim and coworkers reported the potential energy profiles of enzymatic reaction models for wild-type and various mutant $\mathrm{KSI}$ enzymes by using ab initio calculations at the MP2/ $6-31+\mathrm{G}^{*} / \mathrm{B} 3 \mathrm{LYP} / 6-31+\mathrm{G}^{*}$ level ${ }^{10}$ On the basis of the catalytic mechanism described in Scheme 1 , they could successfully explain the mutation effects on the catalytic performance of KSI.

There has been a strong debate on the origin of enzymatic catalysis for a reaction involving an enolization step. ${ }^{\text {"l }}$ The question was whether the electrophilic catalyses could explain the extraordinarily high rate enhancement. In this work, we address this problem by estimating the extent of the decrease in activation free energy due to the operation of two active site acid residues, Tyr14 and Asp99, of KSI. We employ the formate-catalyzed isomerization of 3-butenal, the simplest $\beta, \gamma$-unsaturated carbonyl compound, as an approximate representation of acetate-catalyzed iomerization of 1 in aqueous solution (Scheme 2), which has served as a reference reaction for evaluating the catalytic capability of $\mathrm{KSI}^{1 ?}$
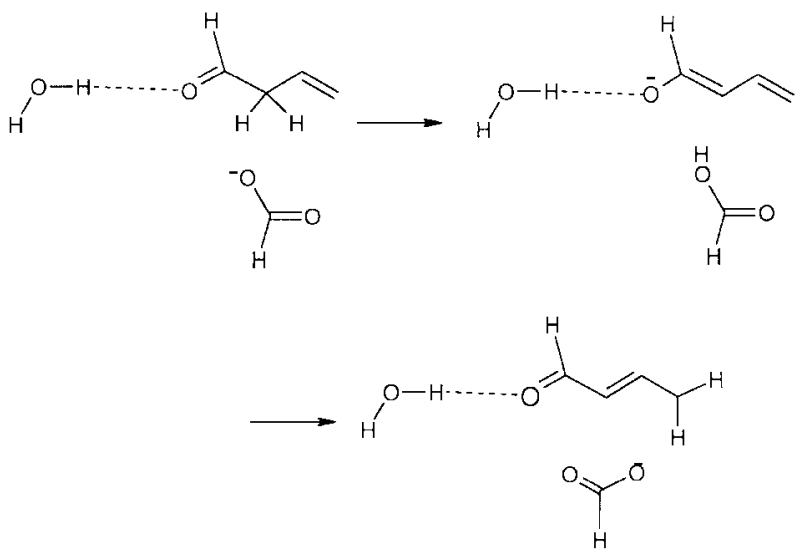

Scheme 2
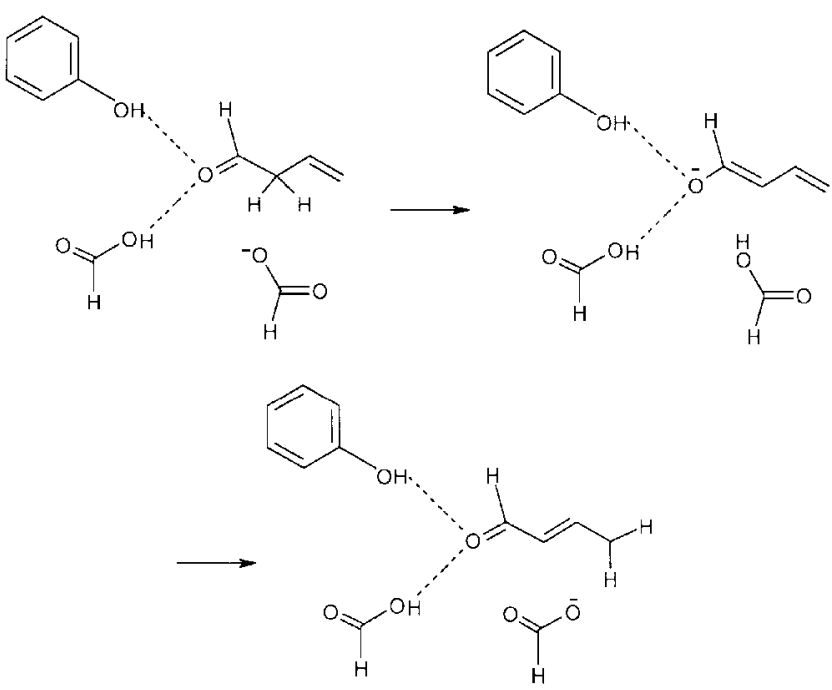

Scheme 3

For this model system, we take into account not only the dielectric medium effect through the polarized continuum model (PCM) ${ }^{13}$ but also the electrophilic catalysis by a solvent water molecule, which has been considered an important aspect of the nonenzymatic reaction in aqueous solution. Indeed, it has been suggested that the transition states and internediates are more stabilized in a nonaqueous environment as in an enzymatic active site than in water. ${ }^{14}$

As an enzymatic reaction model, we consider Scheme 3 in which the roles of general acid catalysts are played by phenol and fomic acid, representing Tyr14 and Asp99, respectively, while formate simulates the action of Asp38.

In contrast to the nonenzymatic case, solvation effects are ignored in this model. This is based on the previous experimental observation that the layer of apolar residues and the ketosteroid substrate would prevent the bulk solvent from accessing the active site of KSI during its catalytic action. ${ }^{7,80}$

In addition to the potential energies, we also evaluate the zero-point vibrational energies and entropic tems for stationary-state structures of both enzymatic and nonenzymatic reaction models to calculate the free energy profiles. By comparing the overall free energies of activation, we estimate the extents of rate enhancement offered by Tyr14 and Asp99. We also examine the variation of geometrical features along intrinsic reaction coordinate (IRC), and compare them with those of the corresponding nonenzymatic reaction to gain some insight into the catalytic strategy of KSI.

There has been a controversy on the mode of catalytic actions of the enzymes that catalyze reactions of carbon acids. It was proposed that the extraordinary efficiency of enzymatic reactions could be attributed to the stabilization of the transition state for proton abstraction by the electrophilic catalysis, which overcomes the kinetic problem of the nonenzymatic reaction with an inherently low rate. ${ }^{16}$ On the other hand, there is also an argument that a thermodynamic difficulty in forming an enolate intermediate is the fundamental problem that should be surmounted by enzymes. ${ }^{1 ?}$ 
By comparing the free energy profiles for the enzymatic and nonenzymatic reaction models under consideration, we will address the issue of which kind(s) of problems can be resolved by catalytic acid residues of KSI.

\section{Computational Methods}

All geometries corresponding to minima and transition states on the two model enzymatic reaction pathways are fully optimized at RHF/ $6-31 \mathrm{G}^{* *}$ level of theory with the latest version of GAMESS code. ${ }^{18}$ These geometry optimizations are performed with the aid of analytically determined gradients and search algorithms of quasi-Newton-Raphson procedure. $^{19}$ The nature of each SCF stationary point is determined by the number of imaginary frequencies that are evaluated by diagonalizing the analytical hessian. Each transition state structure possesses a single negative eigenvalue of the hessian matrix, and the corresponding imaginary vibrational frequency is related to the time scale of the motion that carries the reaction system over the energy barrier. The intrinsic reaction coordinate (IRC) connecting a transition state to the neighboring stable structures is determined by using the Gonzalez-Schlegel second-order (GS2) method $^{20}$ at the same level of theory used in the preceding geometry optimizations.

In order to achieve better prediction of the energetics, post-HF level calculations including the effect of electron correlation are performed at the optimized geometries. These single point calculations are carried out with the $6-31+\mathrm{G}^{* *}$ basis set using the Moller-Plesset second-order perturbation theory (MP2). ${ }^{21}$ The electronic energies computed in this way are then used to calculate the relative free energies in the gas phase $\left(\Delta G_{g}^{\mathrm{u}}\right)$ that are given by

$$
\Delta G_{g}^{\dot{u}}=\Delta E_{e i e:}+\Delta H^{\prime}-T \Delta S
$$

Here, $\Delta H^{\prime}$ denotes the enthalpy change due to thermal motions of the nuclei including the zero-point vibrational energies, and $\Delta S$ is the entropy change. While the electronic energies $\left(E_{\varepsilon / 2 c}\right)$ are evaluated at the MP2/6-31+G** level, the vibrational frequencies used to estimate $\Delta H^{\prime}$ and $\Delta S$ are obtained at the theoretical level adopted in geometry optimizations.

In obtaining the free energy profile for the model nonenzymatic reaction, solvation effects need to be included, since the reference reaction for evaluating the catalytic capability of KSI has been a base-catalyzed isomerization of 1 in aqueous solution. ${ }^{12}$ Therefore, we perform a PCM self-consistent reaction field (SCRF) calculation for the stationarystate structures on the reaction path to compute their solvation free energies $\left(\Delta G^{\text {sorwtion }}\right)$. These single point calculations are performed at the RHF/6-31G** level with dielectric constant 78.15 . The results are then used to estimate relative free energies $\left(\Delta G_{i}^{\dot{v}}\right.$ ) of reactant-state, intermediate, transition states, and product-state structures of the uncatalyzed reaction in solution according to the following equation:

$$
\Delta G_{i}^{\dot{j}}=\Delta G_{g}^{\dot{j}}+\Delta \Delta G^{\text {solination }} \text {. }
$$

\section{Results and Discussion}

Nonenzymatic reaction. We first consider the formateassisted isomerization of 3-butenal in aqueous solution under the general acid catalysis by a solvent water molecule. This is the simplest relevant model for the acetate-catalyzed isomerization of 1 in aqueous solution that has been used as a reference reaction for discussing the enzymatic activity of $\mathrm{KSI}^{1 ?}$ A comparative study of the model nonenzymatic reaction (Scheme 2) and the corresponding enzymatic reaction (Scheme 3) will help us to understand the electronic and geometrical requirements for the exceptional catalytic activity of KSI.

Figure 1 illustrates the variation of fiee energy along the nonenzymatic reaction path as computed by Eq. (2). The energies of the stationary-state structures are measured with respect to the reactant complex (UR).

As indicated in Scheme 2, the first step involves the proton transfer from the $\alpha$-carbon of 3-butenal to formate, leading to the formation of a dienolate reaction intemediate (UINT) with a free energy of $11.9 \mathrm{kcal} / \mathrm{mol}$ above the reactant complex (UR). A kinetic barrier of $13.1 \mathrm{kcal} / \mathrm{mol}$ should be overcome in this step. Then, the reketonization reaction from the unstable dienolate intemediate gives a product complex (UP) that is $4.6 \mathrm{kcal} / \mathrm{mol}$ lower in free energy than UR. The kinetic barrier in this step is predicted to be about $5.3 \mathrm{kcal} /$ mol. The second transition state (UTS2) has higher free energy than the furst transition state (UTS1) by $4.2 \mathrm{kcal} / \mathrm{mol}$. This energetic feature is in good agreement with that observed for acetate-catalyzed isomerization of 1 in aqueous solution. ${ }^{12 b}$ Since the rate constant for the backward transformation from UINT to UR is more than $10^{3}$ times larger

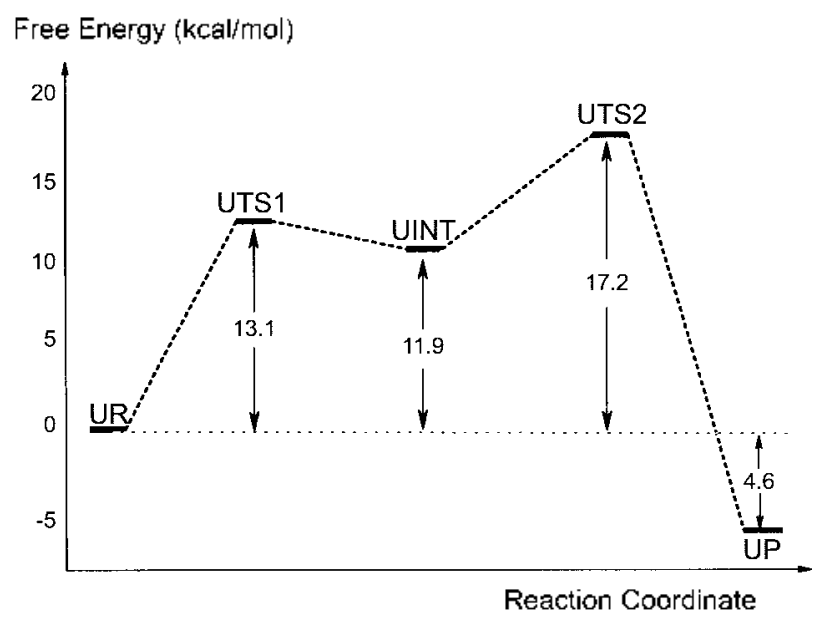

Figure 1. Free energy profile diagram along the intrinsic reaction coordinate of the model nonenzymatic reaction, formate-assisted isomerization of 3-butenal. For each stationary-state structure, the free energy measured from reactant complex (UR) is indicated in units of kcalimol. UTS1. UINT, UTS2, and UP designate the first transition state, reaction intermediate, second transition state, and product complex, respectively. 
than that for the forward transformation into UP, the overall rate constant for the conversion of UR to UP can be approximated as the product of the equilibrium constant for the first step and the rate constant for the second step. Thus, the overall free energy of activation is given by the energy difference between UTS2 and UR, that is $17.2 \mathrm{kcal} / \mathrm{mol}$.

The large energy difference $(11.9 \mathrm{kcal} / \mathrm{mol})$ between UINT and $\mathbf{U R}$ is consistent with previous experimental findings ${ }^{17}$ indicating that the difficulty in forming the dienolate intermediate poses a thermodynamic problem that should be overcome by the catalytic action of KSI. For enzymes like mandelate reacemase that catalyze the enolization reaction of carbon acids, it is known that the reduction of the potential barrier is achieved by utilizing some strong interaction rather than by the general acid-base catalysis. ${ }^{1 l b}$ In the case of KSI, however, it is likely that hydrogen-bond stabilization of the transition state and dienolate intermediate could be a significant catalytic factor because the thermodynamic difficulty of forming the enolate intermediate was found to be less severe for substrates like aldehydes, ketones, and thiolesters than for carboxylic acid and ester substrates. ${ }^{\text {? }}$

We now detail the variation of geometrical properties of the reaction system along the IRC. The stationary-state structures located along the reaction path are shown in Figure 2, together with some selected interatomic distances.

In the reactant-state structure (UR) a negatively charged oxygen $\left(\mathrm{O}_{\mathrm{b}}\right)$ of formate is directed to the hydrogen $\left(\mathrm{H}_{\mathrm{s}}\right)$ attached to the $\alpha$-carbon (C2), forming a weak hydrogen bond with associated $\mathrm{O}_{\mathrm{b}} \cdots \mathrm{H}_{\mathrm{s}}$ distance of $2.370 \AA$. From this complex the first reaction step is initiated by the transfer of $\mathrm{H}_{s}$ from $\mathrm{C} 2$ to $\mathrm{O}_{\mathrm{b}}$. When the $\mathrm{C} 2 \cdots \mathrm{H}_{s}$ distance is elongated from $1.084 \AA$ to $1.422 \AA$, the reaction system reaches the first transition state (UTS1) which is late in terms of $\mathrm{O}_{6}-\mathrm{H}_{5}$ bond formation. The $\mathrm{O}_{0}-\mathrm{H}_{s}$ distance is shortened by $1.157 \AA$ in going from UR to UTS1, which corresponds to $83 \%$ advancement toward the fonnation of the reaction intermediate (UINT in Figure 2). In spite of this significant progress, $\mathrm{C} 1-\mathrm{O}_{5}$ and $\mathrm{C} 3-\mathrm{C} 4$ bond lengths are stretched by only $0.014 \AA$ and $0.009 \AA$, which correspond to $38 \%$ and $47 \%$ progress of the first reaction step, respectively. This implies that the delocalization of negative charge on C2 lags belind the proton transfer, indicating an imbalanced nature of UTS1. Thus, the geometrical features are consistent with the experimentally predicted carbanionic character of the transition states of enolization reactions. ${ }^{2.3}$

A complete transfer of the $\mathrm{H}_{s}$ to $\mathrm{O}_{b}$ leads to the formation of the second energy minimum on the reaction path, UINT, which includes the dienolate intermediate. It is noted that a hydrogen bond between the water molecule and the $\mathrm{O}_{5}$ atom is matured in going from UR to UINT, contributing to the stabilization of the dienolate intennediate. However, the solvent catalysis alone is insufficient to overcome the inherent themodynamic barrier.

From the complex UINT, the second reaction step starts with the approach of formate, in its protonated form, to the $\mathrm{C} 4$ atom, leading to formation of the second transition state (UTS2). In this structure, $\mathrm{H}_{5}$ atom is partially transferred to the $\mathrm{C} 4$ atom with the associated $\mathrm{C} 4 \cdots \mathrm{H}_{5}$ distance of $1.459 \AA$. The reaction system then transforms to the final minimum energy structure (UP), and the model substrate completes the isomerization from $\beta, \gamma$-unsaturated to $\alpha, \beta$-unsaturated carbonyl.

Enzymatic reaction. To estimate the contribution of electrophilic catalysis to the enzymatic rate enhancement of KSI,
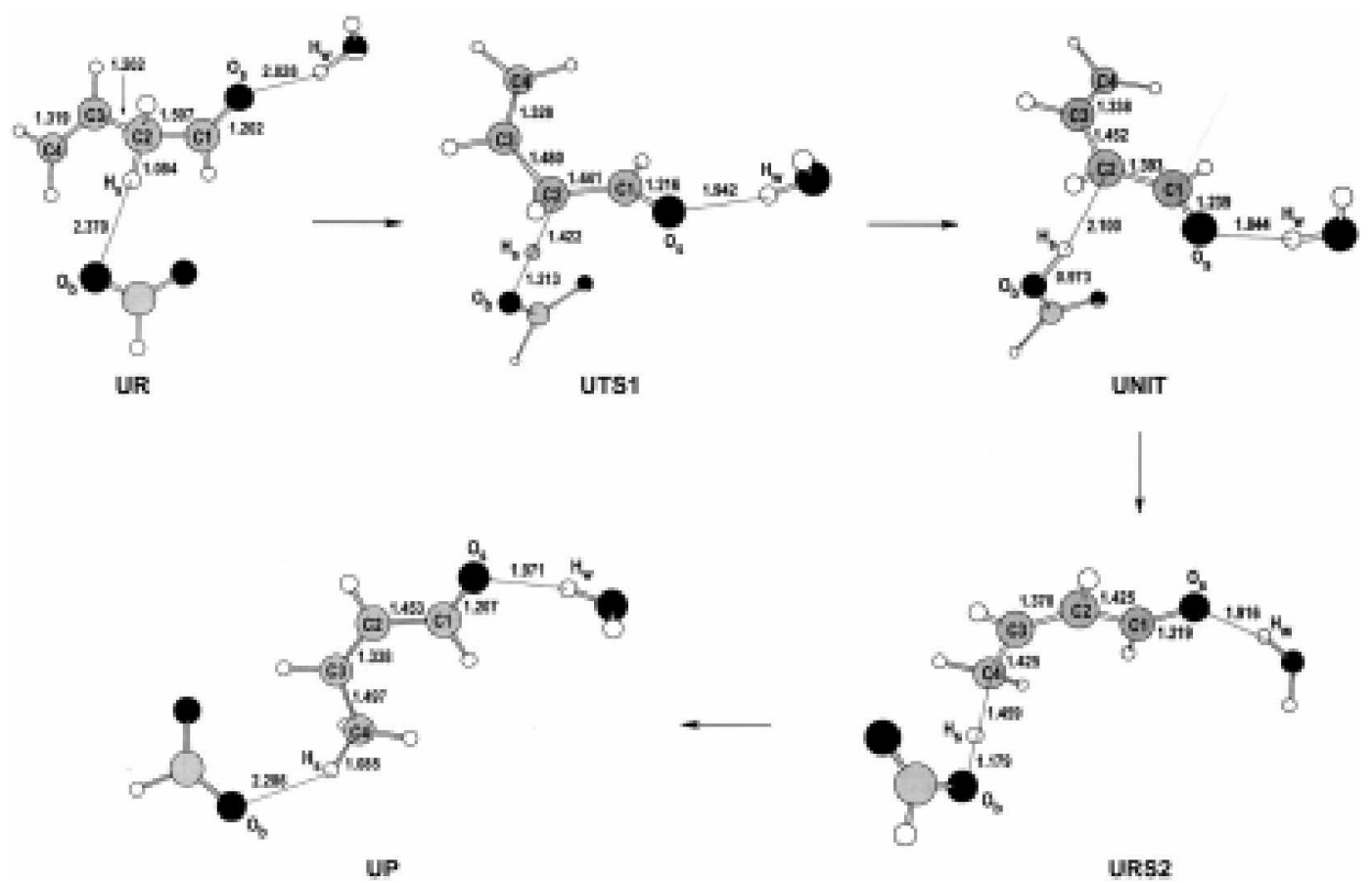

Figure 2. A pictorial description of the reaction pathway for the model nonenzymatic reaction. Some selected interatomic distances are given in $\AA$. 


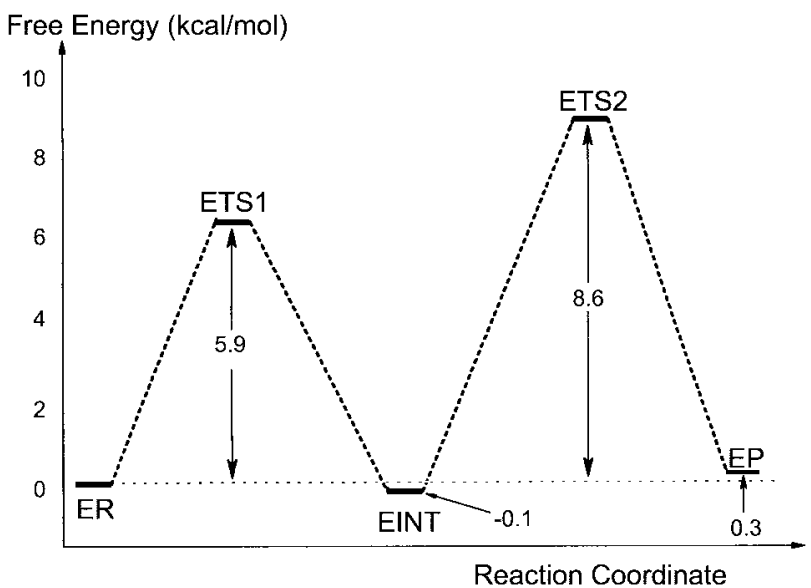

Figure 3. Free energy profile diagram along the intrinsic reaction coordinate of the model enzymatic reaction, formate-assisted isomerization of 3-butenal with the electrophilic catalysis by phenol and formic acid. For each stationary-state structure, the free energy measured from reactant complex (ER) is indicated in units of kcalmol. ETS1, EINT, ETS2, and EP designate the tirst transition state, reaction intenmediate, second transition state, and product complex, respectively.

we have carried out calculations on the formate-catalyzed isomerization reaction of 3-butenal in the presence of phenol and formic acid (see Scheme 3). In this reaction model, formate, phenol, and formic acid correspond to Asp38, Tyr 14, and Asp99 of KSI, respectively. Figure 3 displays the variation of free energy along the reaction coordinate.

First, it is seen that the dienolate intermediate can be dramatically stabilized when complexed with two catalytic acid residues. The stabilization energy for the intermediate (EINT) relative to the nonenzymatic reaction amounts to $12.0 \mathrm{kcal} / \mathrm{mol}$, making the formation of unstable dienolate internediate themodynamically favorable. This indicates that owing to the hydrogen-bond stabilization of the dienolate intermediate by two acid residues, KSI can achieve the reduction of the thermodynamic potential by $-12 \mathrm{kcal} / \mathrm{mol}$ as required for the unexpected acidity observed in enzymatic enolization of a substrate carbon acid. ${ }^{11 \mathrm{~b}}$

The substantial stabilization of the reaction internediate renders the second activation barrier higher than the first one by $2.8 \mathrm{kcal} / \mathrm{mol}$ in the model enzymatic reaction, which is an energetic feature consistent with available experimental data. ${ }^{\text {lnb }}$ Since the rate constant for the backward transformation from EINT to ER is more than $10^{2}$ times larger than that for the forward transformation into EP, the overall rate constant for the conversion of ER to EP is approximately given by the product of the equilibrium constant for the first step and the rate constant for the second step. Then, the overall free energy of activation is the free energy difference between the reactant complex (ER) and the second transition state (ETS2), which is predicted to be $8.6 \mathrm{kcal} / \mathrm{mol}$. This value is lower than that for the nonenzymatic reaction by $8.6 \mathrm{kcal} / \mathrm{mol}$, but the reduction is insufficient to explain the experimental finding. The KSI-catalyzed isomerization reaction of $\mathbf{1} \rightarrow \mathbf{3}$ is about $10^{9}$ times faster than the reference uncatalyzed reaction, ${ }^{12}$ and this rate enhancement corresponds to the reduction of the overall free energy of activation by $12-13 \mathrm{kcal} / \mathrm{mol}$. Apparently, the electrophilic catalyses offered

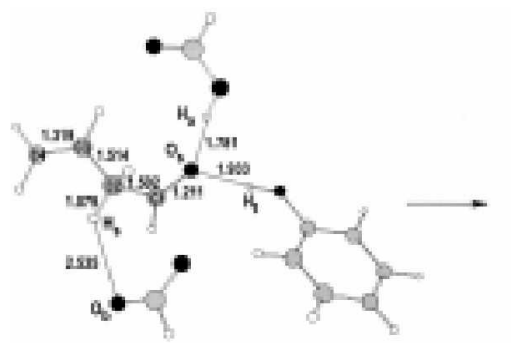

ER

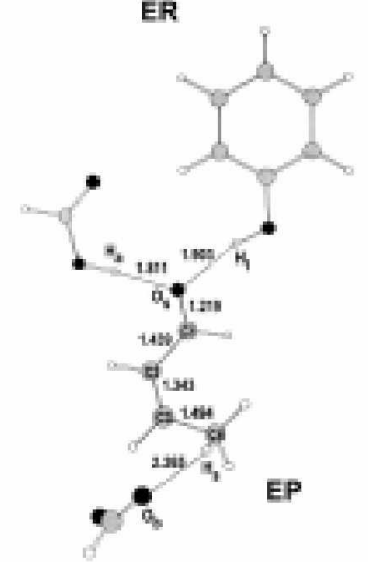

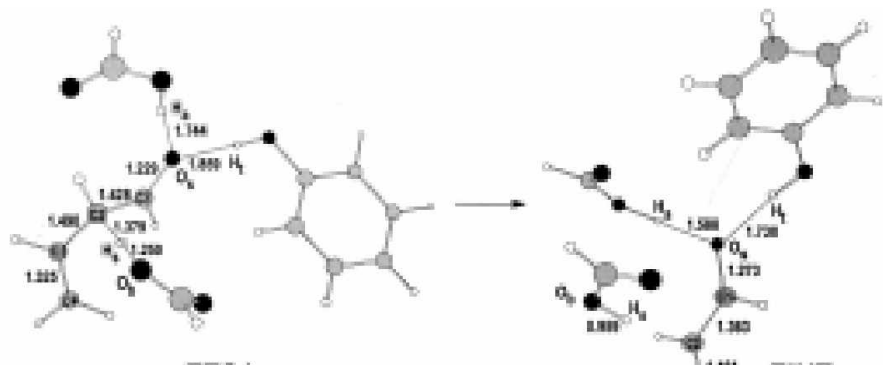

ETS1
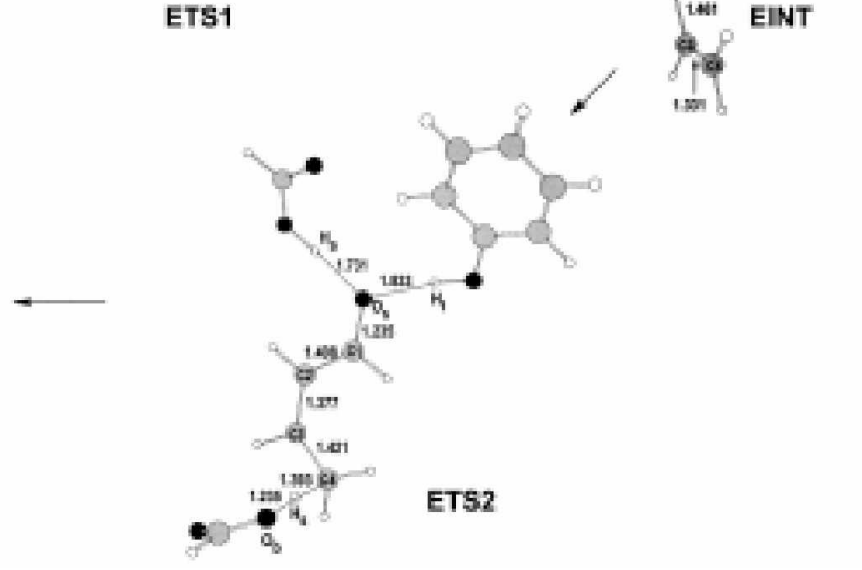

Figure 4. A pictorial description of the reaction pathway for the formate-assisted isomerization of 3-butenal in the presence of phenol and formic acid, which simulates the concerted general acid-base catalysis by the three essential residues of KSI. Some selected interatomic distances are given in $\AA$. 
by the two acid residues make significant contributions to the KSI catalysis, but they are insufficient to fully explain the exceptional catalytic power. This suggests that additional catalytic factors should be involved in the KSI action. In this regard, there is experimental evidence that Phe 101 could also play a significant role in the enzymatic catalysis although the detailed role has not been explained satisfactorily. ${ }^{\text {?3 }}$

We now discuss the geometrical features relevant to the electrophilic catalysis of KSI. Figure 4 displays the structures of energy minima and transition states along the IRC of the enzymatic reaction model.

The first minimum energy structure (ER in Figure 4) corresponds to the substrate complexed with the three catalytic residues. In this structure the hydrogen atoms $\left(\mathrm{H}_{\dagger}\right.$ and $\mathrm{H}_{3}$ ) belonging to the phenolic and carboxylic acid groups of Tyr 14 and Asp99 are directed to the oxygen $\left(\mathrm{O}_{5}\right)$ atom of the substrate, forming relatively weak hydrogen bonds at distances of $1.933 \AA$ and $1.781 \AA$, respectively. Owing to the presence of two additional catalytic residues, interestingly, the $\mathrm{C} 2$ $\mathrm{H}_{5} \cdots \mathrm{O}_{b}$ hydrogen bond becomes weaker compared to that in UR: the $\mathrm{O}_{b} \cdots \mathrm{H}_{\mathrm{s}}$ distance is $2.535 \AA$ in $\mathbf{E R}$, as compared to $2.370 \AA$ in UR.

From the complex ER the reaction starts with the transfer of an $\alpha$-proton $\left(\mathrm{H}_{5}\right)$ from the substrate to the carboxylate group of Asp38 and the simultaneous approach of acid residues, Tyr14 and Asp99, to the $\mathrm{O}_{5}$ atom. The lengthening of the $\mathrm{C} 2-\mathrm{H}_{\mathrm{s}}$ bond from $1.079 \AA$ to $1.379 \AA$ leads to the formation of the first transition state (ETS1) in which both $\mathrm{H}_{\mathrm{t}}$ and $\mathrm{H}_{\mathrm{a}}$ are partially transferred to $\mathrm{O}_{\mathrm{s}}$. In going from $\mathbf{E R}$ to ETS1, the two hydrogen bonds between $\mathrm{O}_{s}$ and catalytic acid residues mature into a stronger form; the associated $\mathrm{O} \cdots \mathrm{H}$ distances shorten from $1.933 \AA$ and $1.781 \AA$ in ER to $1.859 \AA$ and $1.744 \AA$ in ETS1, respectively. This geometrical feature satisfies the libido rile of Jencks ${ }^{24}$ since the full protonation of $\mathrm{O}_{s}$ is likely to be unfavorable in the transition state.

Like UTS1, the transition state ETS1 is late in terms of the proton transfer from the substrate carbon acid to the carboxylate group of Asp38. The $\mathrm{O}_{\mathrm{b}}-\mathrm{H}_{\mathrm{s}}$ distance contracts from $2.535 \AA$ in ER to $1.250 \AA$ in ETS1, which corresponds to $82 \%$ advancement toward the formation of the reaction intermediate. This result is in agreement with the previous experimental finding that proton transfer from the substrate to Asp38 would be well advanced in the first transition state of a KSI-catalyzed reaction. ${ }^{25}$ On the other hand, the lengthening of $\mathrm{Cl}-\mathrm{O}_{s}$ and $\mathrm{C} 3-\mathrm{C} 4$ bonds make $30 \%$ and $50 \%$ progress, respectively, toward the formation of dienolate intermediate, implying that the delocalization of negative charge on $\mathrm{C} 2$ lags behind the proton transfer in the ETS1. This structural feature may be viewed as support for the earlier proposal for the transition state structure in the enolization of 1 by KSI, namely that the carbanionic character would prevail over the enolate one. ${ }^{26}$ Thus, it is likely that the transition state imbalance might also be involved in the KSIcatalyzed isomerization of 1 . As can be inferred from the 7.2 $\mathrm{kcal} / \mathrm{mol}$ decrease in the activation barrier for the first step, however, the barrier enhancing effect of delayed charge delocalization can be negated significantly by the electrophilic catalyses of the two acid residues.

A complete transfer of the $\alpha$-proton from the model substrate to the formate ion carries the reacting system to the second energy minimum (EINT in Figure 4), which corresponds to the dienolate intermediate bound to the active site residues of KSI. We note that protons of acid residues are not fully transferred to the substrate oxygen in EINT. This is in accordance with the earlier proposal of Gerlt and Gassman. Based on the Marcus theory, they argued that partial proton transfer from the acid catalysts to the substrate carbonyl group would be sufficient to explain the enzymatic stabilization of the reaction internediate. ${ }^{13}$ Thus, the reaction intemediate in the KSI-catalyzed reaction has the enolate character rather than the enolic one, as also proposed experimentally by $\mathrm{Wu}$ and coworkers. ${ }^{7}$

It is noteworthy that $\mathrm{H}_{\mathrm{r}}{ }^{\cdots} \mathrm{O}_{s}$ and $\mathrm{H}_{a} \cdots \mathrm{O}_{s}$ hydrogen bonds mature into a much stronger form in going from ER to EINT. Moreover, the magnitude of the strengthening exceeds that of the $\mathrm{H}_{\mathrm{w}} \cdots \mathrm{O}_{\mathrm{s}}$ hydrogen bond in the nonenzymatic case (cf. Figures 2 and 4), which is responsible for the predicted 12.0 $\mathrm{kcal} / \mathrm{mol}$ stabilization of dienolate intermediate in the enzymatic reaction. Indeed, it is well appreciated that the strengthening of the hydrogen bond in enzymatic reactions should be greater than that in the reference uncatalyzed reaction. The energetic and structural features found in this study show that such a catalytic factor is enough to account for the experimentally observed enzymatic stabilization of dienolate internediate. However, the predicted $7.2 \mathrm{kcal} / \mathrm{mol}$ reduction of the first activation barrier is unable to explain the experimental data, according to which the two transition states should be stabilized at least by $11 \mathrm{kcal} / \mathrm{mol}$ in the enzymatic reaction. ${ }^{12}$ This disagreement also suggests the possible involvement of additional catalytic sources in the operation of KSI.

The second reaction step proceeds in a similar way as in the nonenzymatic reaction except that two acid residues stabilize both the second transition state (ETS2) and the product complex (EP) through hydrogen bonds. The mechanistic features of the reketonization step in the real enzymatic reaction are well reflected in the model reaction. Asp38, in its protonated form, delivers the proton $\mathrm{H}_{s}$ to the $\mathrm{C} 4$ atom. and the interactions between $\mathrm{O}_{\mathrm{s}}$ and acid residues are weakened in going from EINT to ETS2. Passing through the second transition state, the reaction system falls into the final minimum energy structure (EP), which corresponds to the reaction product complexed with the catalytic residues. In this structure, the hydrogen bonds between $\mathrm{O}_{s}$ and acid residues become weaker again as compared to those in ETS2.

Finally, we estimate the relative importance of energetic and entropic contributions to the exceptional catalytic power of KSI. Table 1 lists the changes in electronic energy $\left(\Delta E_{\text {elec }}\right)$, thermal energy including the zero-point vibrational energy $\left(\Delta H^{\prime}\right)$, and entropic term $(-T \Delta S)$ along the IRC of the model enzymatic and nonenzymatic reactions.

It is noted that a high value of $\Delta G^{\text {seh sion }}$ for UTS2 relative 
Table 1. Relative contributions of various energetic factor to the changes in free energy along the two reaction paths under consideration. Relative free energies $\left(\Delta G^{0}\right)$ for structures involved in enzymatic and nonenzymatic reaction paths are calculated by using Eq. (1) and Eq. (2), respectively. All energies are given in $\mathrm{kcal} / \mathrm{mol}$

\begin{tabular}{crrrrr}
\hline $\begin{array}{c}\text { Energy minima and } \\
\text { transition states }\end{array}$ & $\Delta E_{\text {ele: }}$ & $\Delta H^{\prime}$ & $-T \Delta S$ & $\Delta \Delta G^{\text {solvation }}$ & $\Delta G^{0}$ \\
\hline Nonenzymatic reaction & & & & & \\
ER & 0 & 0 & 0 & & 0 \\
ETS1 & 8.1 & -4.0 & 1.8 & & 5.9 \\
EINT & -0.4 & -0.6 & 0.9 & & -0.1 \\
ETS2 & 12.0 & -4.2 & 0.8 & & 8.6 \\
EP & 2.1 & -0.2 & -1.6 & & 0.3 \\
Nonenzymatic reactiont & & & & & \\
UR & 0 & 0 & 0 & 0 & 0 \\
UTS1 & 12.7 & -4.0 & 1.8 & 2.6 & 13.1 \\
UINT & 9.7 & -0.4 & 0.9 & 1.7 & 11.9 \\
UTS2 & 12.1 & -4.0 & 2.9 & 6.2 & 17.2 \\
UP & -3.2 & 0.0 & 1.0 & -2.4 & -4.6 \\
\hline
\end{tabular}

to UR is the most significant contributor to the predicted 8.6 $\mathrm{kcal} / \mathrm{mol}$ difference in the free energies of activation between enzymatic and nonenzymatic reactions. This finding clearly indicates that the inclusion of solvation effects should be indispensable for discussing the enzymatic rate acceleration in comparison to the reference uncatalyzed reaction. Interestingly, the potential energy difference between ER and ETS2 is almost equal to that between UR and UTS2, implying that the electrophilic catalyses have little effect on lowering the enthalpy of activation for the nonenzymatic reaction. On the other hand, the overall free energy of activation can be lowered by $2.1 \mathrm{kcal} / \mathrm{mol}$ due to the entropic contribution. This is consistent with the fact that the catalytic residues of KSI can play a role in reducing the entropic penalty for forming the rate-limiting transition state. A similar mechanistic feature has been observed also in the catalytic action of serine proteases ${ }^{28}$ and aspartic proteinases. ${ }^{29}$

\section{Conclusions}

We have estimated the catalytic contribution from two essential active site residues of KSI, Tyr14 and Asp99, by comparing the energetic and structural features of a model enzymatic reaction with those of a corresponding nonenzymatic reaction. Owing to the electrophilic catalytic components, the free energy of activation and the thermodynamic potential of dienolate intermediate are lowered by 8.6 and $12.0 \mathrm{kcal} / \mathrm{mol}$, respectively, in the enzymatic reaction model under consideration. Comparison of these energy values with available experimental data shows that the electrophilic catalyses cannot explain the exceptional rate enhancement of KSI completely, although they can account for the enzymatic stabilization of the dienolate intermediate. Thus, the results of the present study suggest that, to fully resolve the kinetic problem inherent in the reference uncatalyzed reaction, additional catalytic sources may have to be invoked.
The stabilization of the dienolate intermediate is achieved to such an extent that the formation of the intermediate becomes thermodynamically favorable and the reaction rate is controlled by the second step. Considering that the dominant factor destabilizing the rate-limiting transition state is the solvation effect in the reference uncatalyzed reaction, the catalytic activity of KSI is attained by providing a nonaqueous reaction environment as well as efficient catalytic residues. Along the intrinsic reaction coordinate the catalytic residues are positioned such that the entropic penalty for forming the rate-limiting transition state can be minimized. Further study is needed to understand the full details of the catalytic strategy of this proficient enzyme.

Acknowledgment. This work was supported by a grant from the Center for Molecular Catalysis of the Korea Science and Engineering Foundation.

\section{References and Notes}

1. Keeffe, J. R.; Kresge, A. J. In The Chemistry of Enols; Rappoport, Z., Ed.: Wiely: Chichester, England, 1990: Chapter 7.

2. (a) Jencks. W. P. Chem. Rev 1985. 85. 511, (b) Jencks, W. P.; Haber, M. T.; Herschlag. D.; Nazaretian, K. L. J. Am. Chem. Soc. 1986, 108, 479. (c) Saunders. Jr., W, H.; Verth, J. E. V. J. Olg Chem. 1995, 60, 3452. (d) Bemasconi, C. F.: Wenzel, P. J. J. An Chem. Soc. 1996, 118, 11446. (e) Bemasconi, C. F.: Wenzel, P. J.; Keeffe, J. R.: Gronert, S. J. Am. Chem. Soc. 1997, 119, 4008.

3. (a) Murray, C. J; Jencks, W. P. J. Am. Chem. Soc. 1990. 112. 1880. (b) Bemasconi, C. F.; Wenzel, P. J. J. Ant. Chem. Soc 1994. 116. 5405. (c) Bernasconi, C. F.; Panda, M.; Stronach, M. W. $J$. An. Chem. Soc. 1995. 117, 9206. (d) Bemasconi. C. F.; Wenzel, P. J. J. Am. Chem. Soc. 1996, 118, 10494. (e) Nevy, J. B.; Hawkinson, D. C.; Blotny, G.; Yao, X.; Pollack, R. M. J. Am. Chem. Soc. 1997, 119, 12722. (f) Yao, X.; Gold, M. A.; Pollack, R. M.J. An. Chem. Soc. 1999. 121.6220.

4. Radzicka. A.; Wolfenden. R. Science 1995, 267. 90.

5. (a) Kuliopulos, A.; Mildvan, A. S.; Shortle. D.; Talalay. P. Biochemistry 1989, 28, 149. (b) Bounds, P. L.; Pollack, R. M. Biochemistig 1987, 26, 2269. (c) Zawrotny, M. E.; Hawkinson, D. C.: Blotny, G.; Pollack, R. M. Biochemistry 1996, 35, 6438. (d) Viger, A.; Coustal. S.; Marquet. A. J. Am. Chem. Soc. 1981. 103, 451 .

6. (a) Austin, J. C.; Kuliopulos. A.; Mildvan, A. S.; Spiro, T, G. Protein Sci. 1992, 1, 259. (b) Austin, J. C.; Zhto, Q.; Jordan, T.; Talalay, P.; Mildyan, A. S.; Spiro, T. G. Biochemistry 1995, 34 , 4441. (c) Li, Y. K.; Kuliopulos, A.; Mildvan, A. S.: Talalay, P. Biochemistry 1993, 3. 1816. (d) Holman. C. M.; Benisek. W. F. Biochemistiy 1995. 34. 14245. (e) Weintraub, H.; Alfsen, A.; Baulieu. E.-E. Etu, I. Biochem. 1970. 12, 217.

7. Wu, Z. R.: Ebrahimian, S.: Zawrotny, M. E.: Thomburg, L. D.: Perez-Alvarado, G. C.: Brothers, P.: Pollack, R. M.: Summers, M. F. Science 1997, 276, 415.

8. (a) Thomburg, L. D.; Henot, F.: Bash, D. P.; Hawkinson, D. C.; Bartel. S. D.; Pollack, R. M. Biochemistry 1998, 37, 10499. (b) Pollack. R. M.; Thomburg. L. D.; Wu, Z. R.; Summers. M. F. Aich. Biochem. Biophts. 1999, 370. 9. (c) Cho. H.-S.; Choi. G.; Choi, K. Y.; Oh, Y.-H.; Biochemistry 1998, 37, 8325. (d) Choi, G.; Ha, N.-C.: Kim, S. W.: Kim, D.-H.; Park, S.; Oh, B.-H.: Choi, K. Y. Biochemistry 2000, 39, 903 . (e) Kim, D.-H.: Jang, D. S.: Nam, G. H.; Choi, G.; Kim. J.-S.; Ha. N.-C.; Kim, M.-S.; Oh. B.-H.; Choi, K. Y. Biochemistm 2000. 39. 4581.

9. (a) Zhao, Q; Abeygunawardana, C.; Talalay. P.; Mildvan. A. S. Proc. Natl. Acad. Sci. 1996, 93, 8220. (b) Zheo, Q.; Abeygunawardana, C.; Gittis, A. G.; Mildyan, A. S. Biochemistry 
1997, 36. 14616. (c) Massiah, M. A.; Abeygunawardana, C.; Gittis, A. G.; Mildvan. A. S. Biochemistry 1998, 37, 14701.

10. Kim. K. S.; Oh. K. S.; Lee, J. Y. Proc. Natl. Acad Sci. USA 2000. 97,6373

11. (a) Gerlt, J. A.; Gassman. P. G. J. Am. Chem. Soc. 1993, H5, 11552. (b) Guthrie. J. P.; Kluger, R. J. Am. Chem. Soc. 1993, H5, 11569 .

12. (a) Zeng. B.; Pollack, R. M. J. Am, Chem. Soc. 1991, H3, 3838. (b) Hawkinson, D. C.; Eames, T. C. M.; Pollack, R. M. Biochemisty 1991, 30. 10849 .

13. (a) Miertus, S.; Tomasi, J. Chem. Phys. 1982, 65, 239. (b) Mieltus, S.; Scrocco. E.; Tomasi, J. Chem. Phys. 1981, 55, 117.

14. Shan, S.; Herschlag. D. Proc. Natl. Acad Sci. USA 1996, 93. 14474 .

15. As a check on the validity of the enzymatic reaction model we compared the AMl potential energy profile calculated from the present model with that based on a more realistic model, acetatecatalyzed isomerization of 5-androstene-3.17-dione to 4androstene-3,17-dione under the electrophilic catalysis by phenol and acetic acid. The differences in the free energies calculated from the two models for the first transition state, reaction intemediate, second transition state, and product complex are all less than $1.0 \mathrm{kca} 1 / \mathrm{mol}$.

16. (a) Gerlt, J. A.; Gassman. P. G. J. Am. Chem. Soc. 1992, I14, 5928. (b) Gerlt. J. A.; Kozarich. J. W.; Kenyon. G. L.; Gassman. P. G. J.Am Chem Soc 1991, 113,9667.

17. (a) Chiang. Y; Kresge, A. J.; Prnszynski, P.; Schepp. N. P.; Wirz. J. Angew: Chem., Int. Ed. Engl. 1990, 29, 792. (b) Kluger, R.
Chem. Rer: 1990. 90,1151. (c) Thibblin. A; Jencks, W. P. J. Am Chent. Soc. 1979. 101, 4963.

18. Schmidt. M. W.; Baldridge. K. K.; Boatz. J. A.; Elbert, S. T.; Gordon, M. S.; Jensen, J. H.; Koseki, S.; Matsunaga, N.; Nguyen, K. A.: Su, S.: Windus, T. L.: Dupuis, M.: Montgomery, J. A. $J$. Comput. Chem. 1993, 14, 1347.

19. (a) Baker. J. J. Comput. Chen. 1986. 7. 385, (b) Helgaker, T Chen. Phts. Lett. 1991, 182, 305. (c) Bell. S.; Crighton, J. S. J Chen. Phis. 1984. 80, 2464.

20. Gonzalez, C.; Schlegel, B. H. J. Chem. Phys. 1989, 94, 2154.

21. Moller, C.; Plesset, M. S. Phys. Rev 1934, 46, 618.

22. (a) Albery, W. J. J. Chem. Soc.. Faraday Trans. 1982, 78, 1579. (b) Hegarty. A. F.; Jencks, W. P. J. Am. Chem. Soc. 1975, 97, 7188 .

23. Brothers. P. N.; Blotny. G.; Qi. L.; Pollack. R. M. Biochemistn $1995,34,15453$.

24. Jencks, W. P. J. Am. Chem. Soc 1972, 94, 4731

25. Holman, C. M.; Benisek, W. F. Biochemistry 1994, 33, 2672.

26. Xue, L.; Talalay. P.; Mildvan. A. S. Biochenisty 1990, 29, 7491.

27. (a) Ross. A. M.; Whalen. D. L.; Eldin. S.; Pollack, R. M. . A An. Chen. Soc. 1988. H0. 1981. (b) Keeffe, J. R.; Kresge, A. J.; Yin, Y. J. Am. Chem. Soc. 1988, 11 / 1982.

28. (a) Jencks, W. P. Adv. Enz?mol. 1975, 43, 219. (b) Henderson, R. J. Mol. Biol 1970, 54, 341. (c) Rulhlmann, A.: Kukla, D.: Schwager, P.; Bartels, K.; Huber. R. J. Mol. Biol. 1973. 77, 417. (d) Blow, D. M.; Janin, J.; Sweet, R. M. Nathe 1974, 249, 54, (e) Jencks. W. P. Proc. Natl. Acad. Sci. U.S.A. 1981, 78.4046.

29. Park, H.; Suh, J.; Lee, S. J. Am. Chem. Soc. 2000, I22, 3901. 